\title{
BMJ Open Cross-sectional study of the use of antimicrobials following common infections by rural residents in Anhui, China
}

\author{
Jing Chai, ${ }^{\oplus 1,2}$ Caroline Coope, ${ }^{3,4}$ Jing Cheng, ${ }^{1,2}$ Isabel Oliver, ${ }^{3,4}$ Anthony Kessel, ${ }^{5}$ \\ Zhi Hu, ${ }^{1}$ DeBin Wang ${ }^{\circ}$
}

To cite: Chai J, Coope C, Cheng J, et al. Crosssectional study of the use of antimicrobials following common infections by rural residents in Anhui, China. BMJ Open 2019;9:e024856. doi:10.1136/ bmjopen-2018-024856

- Prepublication history and additional material for this paper are available online. To view these files, please visit the journal online (http://dx.doi. org/10.1136/bmjopen-2018024856).

Received 24 June 2018 Revised 29 November 2018 Accepted 31 January 2019

\section{Check for updates}

(c) Author(s) (or their employer(s)) 2019. Re-use permitted under CC BY-NC. No commercial re-use. See rights and permissions. Published by BMJ.

${ }^{1}$ School of Public Health, Anhui Medical University, Hefei, China ${ }^{2}$ School of Health Services Management, Anhui Medical University, Hefei, China ${ }^{3} \mathrm{NIHR}$ Health Protection Research Unit in Evaluation of Interventions, Bristol Medical School, University of Bristol, Bristol, UK

${ }^{4}$ National Infection Service, Public Health England, Bristol, UK

${ }^{5}$ Faculty of Public Health and Policy, London School of Hygiene \& Tropical Medicine, London, UK

Correspondence to

Professor Zhi Hu;

social_capital@sina.com

\section{ABSTRACT}

Objective To describe help seeking behaviour from a medical doctor and antimicrobial use for common infections among rural residents of Anhui province, China. Design A cross-sectional retrospective household survey. Setting 12 administrative villages from rural Anhui, China. Participants 2760 rural residents selected through cluster-randomised sampling using an interviewer administered questionnaire.

Method Logistic regression models were used to estimate associations between exposures (health insurance and antimicrobial-related knowledge), adjusted for confounders (sex, age and education), and help-seeking behaviour from a medical doctor and antimicrobial use following common infections, including acute respiratory tract infections (ARTIs), gastrointestinal tract infections (GTIs) and urinary tract infections (UTIS).

Results In total 2611 (94.6\%) rural residents completed the questionnaire. Help seeking from a medical doctor was highest for ARTIs (59.4\%) followed by GTIs (42.1\%), and UTIs (27.8\%). Around two-thirds (82.3\% for ARTIs, $87.0 \%$ for GTIs and $66.0 \%$ for UTIs) of respondents sought help within 3 days following symptom onset and over three quarters ( $88 \%$ for ARTIs, $98 \%$ for GTIs and $77 \%$ for UTIS) reported complete recovery within 7 days. Of the helpseeking respondents, $94.5 \%$ with ARTI symptoms recalled being prescribed either oral or intravenous antimicrobials (GTIs $81.7 \%$ and UTIs 70.4\%). Use of antimicrobials bought from medicine shops without prescriptions ranged from $8.8 \%$ for GTIs to $17.2 \%$ for ARTIs; while use of antimicrobials leftover from previous illnesses or given by a relative ranged from $7.6 \%$ for UTIs to $13.4 \%$ for ARTIs. Multivariate logistic regression analysis revealed that respondents with a higher antimicrobial-related knowledge score and lack of insurance were associated with lower levels of help-seeking for ARTIs; while respondents with a higher antimicrobial-related knowledge score were less likely to be prescribed either oral or intravenous antimicrobials.

Conclusions Excessive antimicrobial use in the studied primary care settings is still prevalent.

\section{INTRODUCTION}

Antimicrobial resistance (AMR) is a global health problem which claims at least 50000
Strengths and limitations of this study

- The study added new data about the magnitude and determinants of antimicrobial use in China.

- The study distinguished doctor-dominated versus patient-dominated responses towards infections and revealed misperceptions of patients' demand for antimicrobials.

- The study collected data from healthcare users via a household survey while most of the existing research on antimicrobial use in China uses data from medical records or reports by medical caregivers.

- Self-reported antimicrobial use is prone to biases due to recall problems, inability to distinguish antimicrobials from other drugs, conformity to social norms and research expectations.

lives each year across Europe and the USA, with many hundreds of thousands more dying in other parts of the world. ${ }^{1}$ According to the WHO, AMR is threatening our ability to treat common infections in both the community and healthcare settings. ${ }^{2}$ Antimicrobial use, even when appropriate and conservative, contributes to the development of resistance, and inappropriate or excessive use should be avoided. ${ }^{2}$ Numerous studies have reported the relationship between antimicrobial use and the development of resistance. ${ }^{4-7}$ Countries consuming the highest amount of antimicrobials per capita have the highest rates of resistance. ${ }^{8}$

Excessive use of antimicrobials is a widespread problem. Evidence suggests that half of antimicrobial prescriptions are unnecessary or inappropriate for the illness being treated. ${ }^{9-11}$ For instance, it is known that the majority of acute respiratory tract infections (ARTIs) and gastrointestinal tract infections (GTIs) are caused by viruses, ${ }^{12}$ and yet antimicrobial treatment for such infections is common. ${ }^{13-15}$ 
Inappropriate use of antimicrobials and AMR are prevalent in China. Studies published from 2009 to 2013 found that over half of outpatients and around $70 \%$ of inpatients of hospitals in China were prescribed one or more antimicrobials. ${ }^{16-21}$ The problem was even more acute in primary care settings, particularly in rural areas. It had been estimated that, in the same period, $70 \%-90 \%$ of patients visiting village clinics with symptoms of respiratory tract infection were prescribed antimicrobials. ${ }^{22-24}$ Widespread overuse and misuse of antimicrobials parallels rapid growth of AMR in the nation. ${ }^{25-27}$ Comparative studies on the patterns of AMR between different countries indicate that China has one of the highest levels and the fastest growth rates of AMR. ${ }^{26} 28-32$

The antimicrobial use/AMR problem in China has been attributed to a variety of factors. ${ }^{26}{ }^{33-36}$ China has a long history of nearly free consultations in which most patients only pay for prescribed medicines, making prescriptions an important source of revenue for care providers. These remuneration mechanisms have provided a perverse financial incentive for healthcare practitioners resulting in excessive prescribing of antimicrobials, ${ }^{334}$ particularly in the lower levels of the health system. Other determinants of antimicrobial use include expectations and beliefs about antimicrobials among patients ${ }^{35} 36$; diagnostic uncertainty, prescribing habits and misperceptions among clinicians' about service-seekers expectations to receive antimicrobials. ${ }^{37-39}$

China has witnessed fundamental reforms of its healthcare system during the past decade. In 2003, the Chinese government introduced a new rural cooperative medical insurance system available to rural residents at a relatively small per capita annual cost. By 2009 , almost $96 \%$ of rural residents were covered by this insurance. Starting in 2011, the central government implemented a nation-wide Special Antimicrobials Use Rectification programme. ${ }^{40}$ This programme included antimicrobial stewardship in hospitals, AMR monitoring systems, mandatory negative lists of clinical conditions and antimicrobial prescription limits. ${ }^{42} 43$ There is some evidence from county and tertiary hospitals of reductions in antimicrobial use following these initiatives. ${ }^{44-46}$ However, little is known about the effects of these reforms and programmes on antimicrobial use in primary care settings, particularly those in resourcepoor rural areas.

Our study aimed to describe current antimicrobial use and help-seeking from a medical doctor for three common infections, including ARTIs, urinary tract infections (UTIs) and GTIs, among rural residents in Anhui province, China to help inform future interventions aimed at reducing AMR. Most previous studies examining antimicrobial use in rural China were based on extracted data from incomplete service logs preserved at primary care settings or exit surveys of patients visiting these caregivers. ${ }^{47}$ Studies using community samples in China are few, and it is hoped that the findings of this survey will add to this literature and inform future policy reforms and interventions both in Anhui province and other rural settings in China.

\section{METHODS}

\section{Study design and population}

Participants were recruited using a stratified-cluster randomised sampling approach targeting rural residents of Anhui province. Anhui is one of the three self-selected pilot provinces in China and as such has been proactive in implementing the New Health System Reformation which includes optimisation of antimicrobial use. Being the baseline survey of a pilot intervention supported by the UK-China Strategic Prosperity Fund, the sample size was determined by the need to detect difference in antimicrobial use between intervention and control arms. A sample size of 2760 was required to identify $(\mathrm{n}=140)$ cases of UTIs (the lowest incidence among ARIs, GTIs and UTIs) based on empirical estimates of the infections (UTIs $=5 \%$ ), use of antimicrobials following the infection (45\%) and assumed effectiveness of the intervention (reduction in antimicrobial use by $30 \%$ ) adjusted for a $90 \%$ response rate. Participants were randomly selected from 12 administrative villages via a four step process. Step 1 classified all the counties in Anhui province into southern, northern and middle areas. Step 2 randomly selected four counties from each area $(n=12)$ and then one township from each of the counties and one administrative village from each of the townships. Step 3 randomly selected 230 households from each of the 12 administrative villages. Step 4 consisted of randomly selecting one eligible member from each household. The randomisation used a web-based aid, a simple self-developed webpage which had an input box for entering the last number (say, n) of order (in terms of age) of eligible members within the household which randomly selecting a number between 1 and $n$. Eligibility criteria included men and women who were: (a) living in the sampled village when the survey was conducted, (b) aged 18 years or over and (c) deemed able to answer the survey questions.

\section{Questionnaire}

A structured questionnaire was developed to collect information on the prevalence of symptoms of common infections, help-seeking from medical doctors in any setting and recall of antimicrobial prescription for those infections and other potential determinants (online supplementary Appendix 1). An annual rate (R1) of experience of possible ARTIs was determined based on self- reported symptoms. Rates of self-reported symptoms of GTIs (R2) and UTIs (R3) in the past 3 months were also calculated. Here R1 (or R2 or R3) equals the number of respondents who had reported symptoms of ARTIs (or GTIs or UTIs) in the past year (or 3 months) divided by the total number 
of respondents. Self-medication was defined as the use of medicines without prescription including those: (a) bought from a pharmacy or medicine shop, (b) leftover from previous illnesses or given by relatives. An antimicrobials-related knowledge score was calculated using responses to a selected subset of tailored questions related to AMR. Responses to these questions were scored, post-hoc, as incorrect (score $=0$ ) or correct (score $=1)$. An antimicrobials-related knowledge score was derived by summing all of the scores for the knowledge questions with a higher score indicative of better knowledge about antimicrobials (for detailed scoring system, please see online supplementary appendix 2). Data were also collected on factors identified a priori as potential confounders including sex, age, education and health insurance cover (new rural cooperative medical system, other health insurance systems).

\section{Data collection}

Data collection took place from 22 July to 11 August 2015. The structured questionnaire was administered face-toface by selected students from Anhui Medical University at the households of the respondents. Twelve students were divided into two equal teams and led by a quality supervisor, with each team conducting data collection at six study villages consecutively. The study used faceto-face interviews with residents following informed consent. Data collection took three to 4 days per village and each interview took about $15 \mathrm{~min}$. Measures taken to ensure data quality included: (a) piloting and revision of the questionnaire, (b) training and examination of field data collectors (including questionnaire administration and taking informed consent), (c) daily checks by quality supervisors of all the questionnaires completed during the day, (d) retest of a $5 \%$ randomly selected sample of subjects and (e) feedback of errors found via the daily checks and retests.

\section{Data management and analysis}

Questionnaire responses were double-entered into a database using EPI DATA V.3.1 and then extracted and analysed using SPSS V.10.01 and Microsoft Excel 2013. Data analysis comprised descriptive estimations including non-parametric tests of association (Kruksal-Wallis) and multivariate logistic regression modelling to assess factors associated with help-seeking from a medical doctor, use of prescribed oral antimicrobials, use of prescribed intravenous antimicrobials and use of prescribed oral or intravenous antimicrobials or both (oral/intravenous antimicrobials) adjusted by sex, age (years), education. Cases with missing data were excluded from the data analyses.

\section{Patient and public involvement}

Development of the research questions and outcome measures were informed by qualitative interviews and pilot tests with a purposively selected sample of rural residents and village doctors within the study site communities in Anhui province. Summary reports about the study results will be disseminated to the participating individuals and communities through relevant local websites, newspapers and workshops.

\section{RESULTS}

Sociodemographic characteristics and reported symptoms of common infections

Of the 2760 rural residents sampled, 2611 (95\%) completed the questionnaire. Participants had a mean age of 53 years, ranging from 18 to 95 years old (table 1). Female rural residents made up $60 \%$ of the sample. One-third $(n=865)$ of the sample had no formal education and $2558(98 \%)$ had health insurance. A third $(n=886)$ of respondents had an antimicrobials-related knowledge score of zero, with only 317 (12.1\%) participants scoring three or above (maximum score 6). A large proportion of respondents reported having symptoms of ARTI in the past year $(\mathrm{n}=2223 ; 85 \%)$, with smaller number, 425 $(16 \%)$ and $133(5 \%)$ reporting GTI and UTI symptoms in the past 3 months, respectively.

\section{Help-seeking from a medical doctor following reported symptoms of common infections}

Of the respondents who had reported ARTI symptoms in the past year, $1319(59.4 \%)$ reported seeking help from a medical doctor in response to the symptoms, compared with $28 \%$ of respondents reporting GTI symptoms and $42 \%$ of those reporting UTI symptoms, in the past 3 months (table 2). The proportion of respondents with symptomatic infections who sought help from a medical doctor within 3 days of first experiencing symptoms varied (82\% for ARTIs, $87 \%$ for GTIs and $66 \%$ for UTIs). Of those participants who had recovered from the reported infection when the survey was conducted, over three quarters $(88 \%$ for ARTIs, $98 \%$ for GTIs and $77 \%$ for UTIs) reported complete recovery within 7 days. The median recovery period for ARTI type illness was 4 days, compared with 2 days for GTIs and 4 days for UTIs. Kruskal-Wallis test revealed mixed differences in recovery period for the three categories of infections between participants who had sought help from a doctor and those who had not. Respondents who had sought medical help reported a longer recovery period for ARTIs than those who had not $(\mathrm{p}<0.001)$ but the recovery period was shorter for GTIs $(p<0.001)$; while no significant difference in recovery time was found for UTIs $(p=0.073)$. Online supplementary appendix 3 gives details of helpseeking from a medical doctor for perceived symptoms of ARTIs by different subgroups. The proportion of respondents with ARTI symptoms who reported help-seeking from a medical doctor increased by age-group $(\mathrm{OR}=1.23$, 95\% CI: 1.16 to 1.31 ), but decreased with years of education ( $\mathrm{OR}=0.72,95 \%$ CI: 0.66 to 0.78$)$. These trends were consistent for males and females. The time-lag between ARTI onset and help-seeking from a medical doctor did not vary by age or education. A higher proportion of 
Table 1 Sociodemographic characteristics and reported symptoms of common infections among rural residents ( $\mathrm{n}=2611$ )

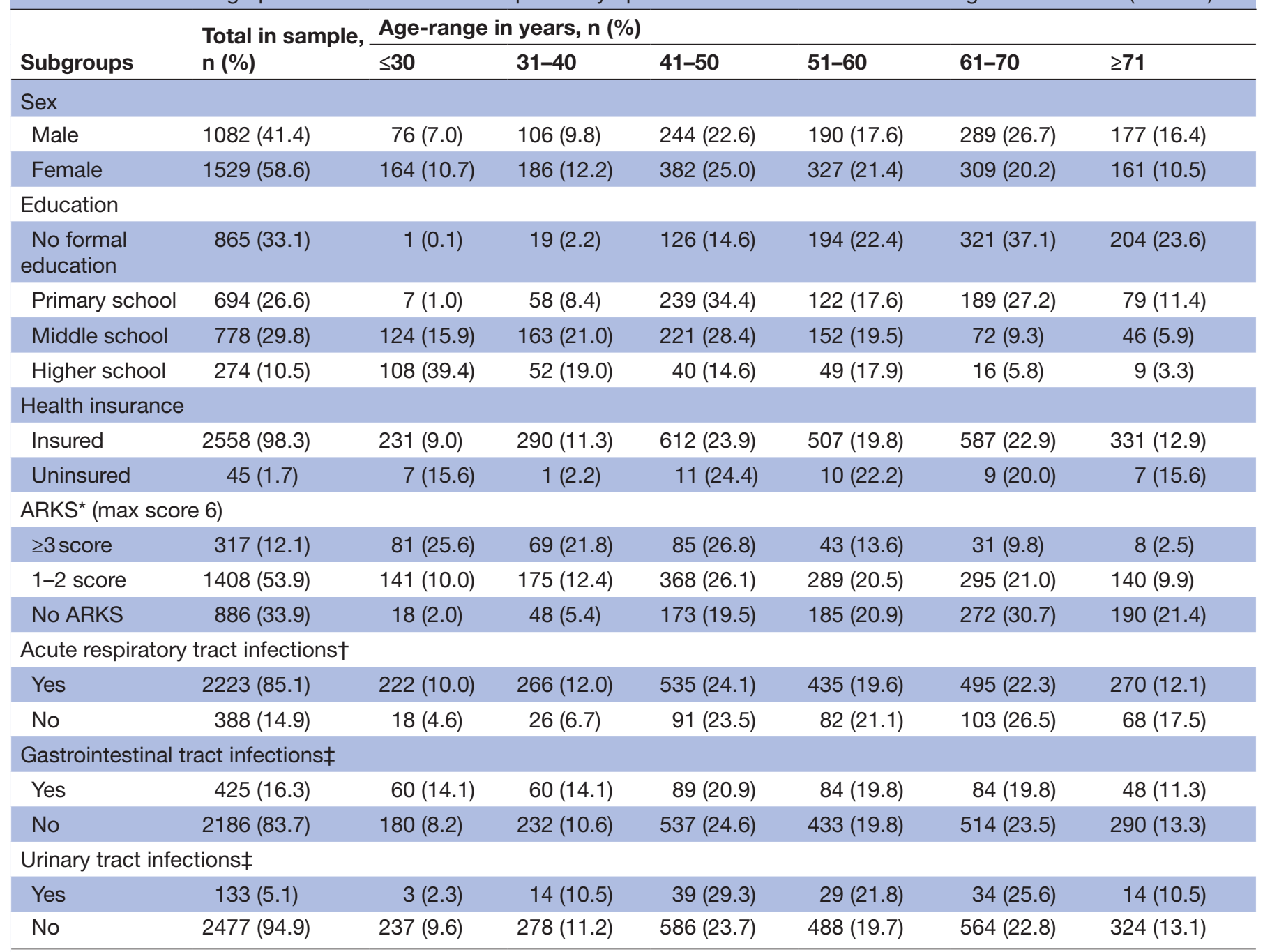

*Antimicrobials-related knowledge score.

†Most recent episode within the past 12 months.

fEpisode within the past 3 months.

ARKS, antimicrobial-related knowledge score.

respondents recalled seeking help from a medical doctor on days 2 and 3 following illness onset for all age and education subgroups.

\section{Use of prescribed antimicrobials after infections}

Of the respondents who recalled seeking help from a medical doctor for their ARTI symptoms, 1059 (80\%) reported visiting a village clinic, 176 (13\%) visited a township health centre, $60(5 \%)$ a county hospital and $29(2 \%)$ a higher level hospital. Of the respondents who sought medical help for an ARTI, 1051 (95\%) said that they had been prescribed oral, intravenous antimicrobials or both (table 3). The proportion of respondents who recalled being prescribed antimicrobials for a GTI or UTI was slightly lower at $82 \%$ and $70 \%$, respectively. Oral antimicrobials were more frequently prescribed than intravenous antimicrobials. There was no statistical evidence of an association between timing of help-seeking and being prescribed antimicrobials.

\section{Self-medication with antimicrobials following infections}

Three-hundred and fifty-four (17\%) people reported self-medication with antimicrobials for ARTIs, $36(9 \%)$ for GTIs and 22 (17\%) for UTIs, respectively (table 4 ). Reported self-medication with antimicrobials left-over from previous illnesses or given by relatives to treat these symptoms was $290(13 \%)$ for ARTIs, 43 (11\%) for GTIs and $10(8 \%)$ for UTIs. The majority of customers to medicine shops bought antimicrobials, being 354 out of $658(63 \%)$ customers with ARTIs, 36 out of $83(51 \%)$ customers with GTIs and 22 out of $33(73 \%)$ customers with UTIs.

\section{Determinants of help-seeking from a medical doctor and antimicrobials use}

Table 5 and online supplementary appendix 4 present the results of our descriptive analysis and multivariate logistic regression modelling, respectively, aimed at exploring determinants of help-seeking from a medical 
Table 2 Help-seeking from medical doctor following reported symptoms of selected common infections

\begin{tabular}{|c|c|c|}
\hline ARTIs* $^{*}(n=2223)$ & $\begin{array}{l}\text { Gastrointestinal tract } \\
\text { infections (GTIs)† ( } n=425)\end{array}$ & UTIs $\$(n=133)$ \\
\hline$\%$ & $\%$ & $n$ \\
\hline
\end{tabular}

Service use

(1) Help-seeking from a medical doctor (Q2.3, Q2.21 and Q2.37)

$\begin{array}{lrrrrrrr}\text { Yes } & 1319 & 59.4 & 118 & 27.8 & 56 & 42.1 \\ \text { No } & 902 & 40.6 & 307 & 72.2 & 77 & 57.9 \\ \text { Not stated } & 2 & \text { NA } & 0 & \text { NA } & \text { NA }\end{array}$

(2) Days until complete recovery after onset of symptoms (Q2.18, Q2.34 and Q2.50)

\begin{tabular}{|c|c|c|c|c|c|c|}
\hline Day 1 & 52 & 2.4 & 98 & 25.9 & 6 & 4.7 \\
\hline Day 2 & 262 & 12.3 & 114 & 30.2 & 18 & 14.1 \\
\hline Day 3 & 520 & 24.4 & 65 & 17.2 & 19 & 14.8 \\
\hline Day 5 & 167 & 7.8 & 12 & 3.2 & 5 & 3.9 \\
\hline Day 6 & 64 & 3.0 & 4 & 1.1 & 1 & 0.8 \\
\hline Day 8+ & 231 & 10.9 & 7 & 1.9 & 21 & 16.4 \\
\hline Not yet recovered & 167 & 7.8 & 40 & 10.6 & 37 & 28.9 \\
\hline Not stated & 94 & NA & 47 & NA & 5 & NA \\
\hline
\end{tabular}

(3) Days until first help-seeking from a medical doctor after onset of symptomsł (Q2.6, Q2.24 and Q2.40)

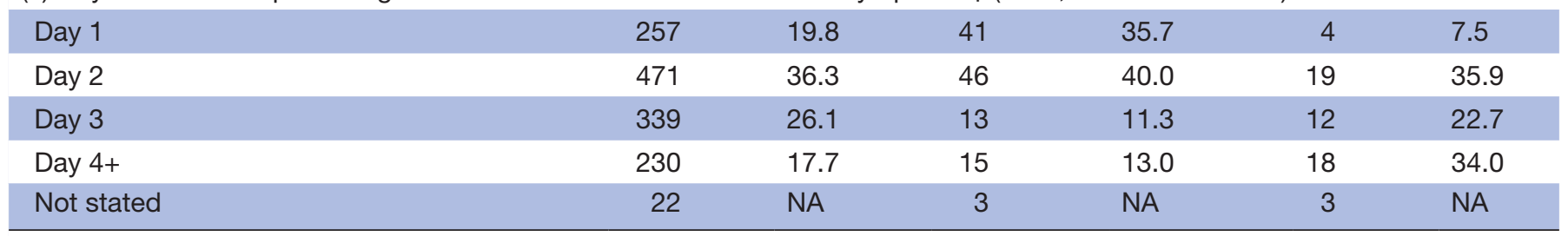

${ }^{*}$ Most recent episode within the past 12 months.

†Episode within the past 3 months.

fIncludes only those who stated 'yes' to use of professional healthcare service.

ARTI, acute respiratory tract infection; NA, not applicable; $Q$ plus a number, the reference number of question used in the questionnaire; UTI, urinary tract infection.

doctor at village clinic, township health centre or higher level service, and determinants of prescribing of oral/ intravenous antimicrobials, oral antimicrobials only and intravenous antimicrobials only following an ARTI. We did not explore factors associated with help-seeking from a medical doctor for GTIs and UTIs due to the relatively small number of cases. The descriptive analysis showed statistically significant differences in these healthcare behaviours between groups with different sex, age, education, insurance and knowledge status. After controlling for a priori confounders (age, sex and education). Helpseeking from a medical doctor was inversely associated to the antimicrobial-related knowledge score with people with a higher knowledge score having a lower odds of help-seeking from a medical doctor in response to their ARTI. There was no association between antimicrobial-related knowledge score and reported prescription of oral or intravenous antimicrobials. However, respondents with a higher antimicrobial-related knowledge score (three or more) were less likely to be prescribed oral/intravenous antimicrobials compared with those with a score of zero (OR=0.32, 95\% CI: 0.13 to 0.78 ). Age displayed a negative association with prescribed oral antimicrobial use $(\mathrm{OR}=0.81,95 \% \mathrm{CI}: 0.71$ to 0.93$)$ but a positive relationship with prescribed intravenous antimicrobial use $(\mathrm{OR}=1.21,95 \% \mathrm{CI}: 1.10$ to 1.33$)$. Health insurance was associated with help-seeking from a medical doctor (OR=0.33, 95\% CI: 0.17 to 0.66$)$. Uninsured respondents had a lower odds of help-seeking from a medical doctor at village clinics $(\mathrm{OR}=0.17,95 \% \mathrm{CI}: 0.06$ to 0.51$)$ but a higher odds of help-seeking from a medical doctor from township or higher tier facility (OR=5.90, 95\% CI: 1.99 to 17.47) compared with insured respondents.

\section{DISCUSSION}

\section{Key findings}

This study has provided an important insight into helpseeking from a medical doctor for common infections in rural China. Symptoms of infections were commonly reported and most people sought help soon after developing symptoms. The majority of people seeking help from 
Table 3 Reported use of prescribed antimicrobials for common infections following help-seeking from a medical doctor

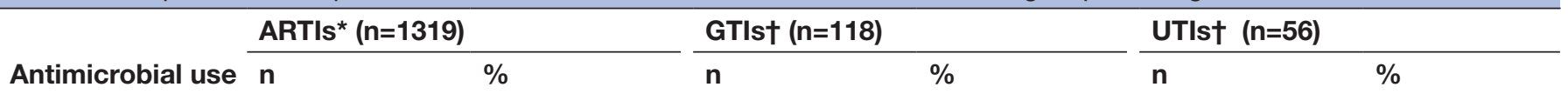

(1) Respondents with clear memory of receiving prescribed oral antimicrobials (Q2.7, Q2.25 and Q2.41)

\begin{tabular}{|lrrrrrl} 
Yes & 638 & 72.7 & 34 & 49.3 & 32 & 74.4 \\
No & 240 & 27.3 & 33 & 50.7 & 11 & 25.6 \\
\hline Not clear & 409 & NA & 43 & NA & 12 & NA \\
\hline Not stated & 32 & NA & 8 & NA & 1 & NA
\end{tabular}

(2) Respondents with clear memory of receiving an intravenous antimicrobial (Q2.10, Q2.27 and Q2.43)

\begin{tabular}{|lcccccc|}
\hline Yes & 705 & 54.7 & 48 & 42.5 & 12 & 22.6 \\
\hline No & 584 & 45.3 & 65 & 57.5 & 41 & 77.4 \\
\hline Not clear & 17 & NA & 3 & NA & 3 & NA \\
\hline Not stated & 13 & NA & 2 & NA & 0 & NA \\
\hline (3) Respondents & with clear memory & of receiving & oral/intravenous antimicrobials (Q2.7, Q2.10, Q2.25 Q2.27, Q2.41 and Q2.43) \\
\hline Yes & 1051 & 94.5 & 67 & 81.7 & 38 & 70.4 \\
\hline No & 61 & 5.5 & 15 & 18.3 & 16 & 29.6 \\
\hline Not clear & 197 & NA & 32 & NA & 1 & NA \\
\hline Not stated & 10 & NA & 4 & NA & 1 & NA \\
\hline
\end{tabular}

(4) Prescribed oral/intravenous antimicrobials by days after onset of symptoms when the first professional service seeking took placeł (Q2.6, Q2.7, Q2.10, Q2.24, Q2.25 Q2.27, Q2.40, Q2.41 and Q2.43)

\begin{tabular}{lrrrrrr} 
Day 1 & 178 & 91.8 & 20 & 80.0 & 1 & 25.0 \\
Day 2 & 383 & 93.9 & 27 & 79.4 & 14 & 77.8 \\
Day 3 & 286 & 96.0 & 8 & 88.9 & 8 & 66.7 \\
Day 4+ & 189 & 95.9 & 12 & 85.7 & 13 & 76.5 \\
Not stated & 15 & NA & 0 & NA & 2 & NA \\
\hline
\end{tabular}

*Most recent episode within the past 12 months.

†Episode within the past 3 months.

łIncludes only those who stated 'yes' to clearly remembering receiving oral or intravenous antimicrobials or both; oral/intravenous antimicrobials mean oral or intravenous antimicrobials or both.

ARTI, acute respiratory tract infection; GTI, gastrointestinal tract infection; NA, not applicable; Q plus a number, the reference number of question used in the questionnaire; UTI, urinary tract infection.

village clinics for ARTI (94\%) recalled being prescribed either oral or intravenous antimicrobials or a combination of both. The reported antimicrobial prescription rates for GTIs and UTIs were lower than that for ARTIs but were still very high at $82 \%$ and $70 \%$, respectively. We found evidence that receiving a prescription intravenous antimicrobials was associated with antimicrobial-related knowledge score and older age. Self-medication with antimicrobials bought from a medicine shop without prescription was reported by nearly $17 \%$ of people for ARTIs, $9 \%$ for GTIs and $17 \%$ for UTIs; while self-medication with antimicrobials left-over from previous illness, or given by relatives, was reported by $13 \%$ of respondents for ARTIs, $11 \%$ for GTIs and $8 \%$ for UTIs. Greater knowledge about antimicrobials and higher level of educational attainment were associated with lower levels of help-seeking from a medical doctor which suggests better education may reduce unnecessary attendances. As expected, lack of insurance was also associated with a lower odds of helpseeking from a medical doctor.
Implications in context of other research and for policy

The study findings have important implications. The very high reported rate of antimicrobial prescriptions in our study indicates that prescription rates remain high in rural areas and contradicts a common belief among policy-makers in China that overuse and misuse of antimicrobials is being brought under control as a result of the nationwide Special Antimicrobials Use Rectification Program (initiated in 2011) and the New Health System Reforms. ${ }^{44-46}$ Rates of antimicrobial prescribing at primary care settings in European countries range from $10 \%$ to $52 \%$ for respiratory tract infections, from $3 \%$ to $22 \%$ for genitourinary infections and from $1 \%$ to $55 \%$ for other infections. ${ }^{49}$ Given that about $57 \%$ of China's vast population lives in rural areas, and over $70 \%$ of antimicrobial prescriptions occur at primary care settings ${ }^{50}{ }^{51}$ there is a clear need for policies and interventions focused primarily on inappropriate antimicrobial use in primary care settings and communities. This is further supported by the finding of no apparent 
Table 4 Use of self-medication with antimicrobials for suspected infections

\begin{tabular}{|c|c|c|c|c|c|c|}
\hline \multirow[b]{2}{*}{ Self-medication } & \multicolumn{2}{|c|}{ ARTIs* $^{*}(n=2223)$} & \multicolumn{2}{|c|}{ GTIs† $(n=425)$} & \multicolumn{2}{|c|}{ UTIs† $(n=133)$} \\
\hline & $\mathbf{N}$ & $\%$ & $\mathbf{N}$ & $\%$ & $\mathbf{N}$ & $\%$ \\
\hline \multicolumn{7}{|c|}{$\begin{array}{l}\text { (1) Respondents who bought medicines for suspected } \\
\text { infection without prescriptions (Q2.14, Q2.30 and Q2.46) }\end{array}$} \\
\hline Yes & 658 & 29.8 & 83 & 19.8 & 33 & 24.8 \\
\hline No & 1551 & 70.2 & 337 & 80.2 & 100 & 75.2 \\
\hline Yes & 354 & 62.5 & 36 & 51.4 & 22 & 72.7 \\
\hline No & 212 & 37.5 & 34 & 48.6 & 6 & 27.3 \\
\hline Not stated & 92 & NA & 13 & NA & 5 & NA \\
\hline Yes & 354 & 16.7 & 36 & 8.8 & 22 & 17.2 \\
\hline No & 1763 & 83.3 & 371 & 91.2 & 106 & 82.8 \\
\hline Not stated & 106 & NA & 18 & NA & 5 & NA \\
\hline \multicolumn{7}{|c|}{$\begin{array}{l}\text { (3) Use of antimicrobials leftover from previous illness or } \\
\text { given by relatives (Q2.17, Q2.33 and Q2.49) }\end{array}$} \\
\hline Yes & 290 & 13.4 & 43 & 10.8 & 10 & 7.6 \\
\hline No & 1872 & 86.6 & 356 & 89.2 & 122 & 92.4 \\
\hline Not stated & 61 & NA & 26 & NA & 1 & NA \\
\hline
\end{tabular}

*Most recent episode within the past 12 months.

†Episode within the past 3 months.

†Includes only those who stated 'yes' to buy medicines for suspected infection without prescriptions.

ARTI, acute respiratory tract infection; GTI, gastrointestinal tract infection; NA, not applicable; $Q$ plus a number, the reference number of question used in the questionnaire; UTI, urinary tract infection.

association between antimicrobial prescribing and days to service seeking after ARTIs.

The study also highlights the need for action to reduce self-medication with antimicrobials leftover from previous illnesses or given by relatives or bought over-the-counter without prescriptions. Self-medication of antimicrobials is very common worldwide.$^{52}$ It has been associated with the risk of inappropriate antimicrobial use which predisposes patients to drug interactions, masks symptoms of underlying disease and promotes the development of microbial resistance. ${ }^{52}$ The reported high level of self-medication with left-over antimicrobials from previous illnesses is likely to be indicative of both overprescription by clinicians, and poor compliance by patients. While reasons underlying the use of self-obtained antimicrobials may be complex, our study identified higher use of reported self-medication in the younger and more educated groups which is in line with previous studies ${ }^{53}$ despite policies which ban the sale of antimicrobials without prescriptions. Future interventions to increase knowledge about appropriate use of antimicrobials should include information about the harms of self-medication.

There may be benefit from differentiated strategies in tackling antimicrobial use behaviours. Our finding of an association between sex, education, health insurance and knowledge of antimicrobials with help-seeking from a medical doctor rather than use of prescribed antimicrobial may suggest the former is dominated by patient-side factors, while the latter, may be more influenced by physician-side factors which were not measured in this study. Similarly, the variations in help-seeking from a medical doctor for ARTIs (59\%), UTIs (42\%) and GTIs (28\%) may reflect the severity and pattern of progression of symptoms of the different infections with patients with acute and aggravating or persisting symptoms being more likely, than those with milder and diminishing symptoms, to seek professional healthcare. Although we did not investigate the reasons people chose either to seek or not to seek professional help, it would be useful to explore this in further depth in future research.

Our findings also point to a need for understanding and tackling antimicrobial use and resistance in a socioculturally sensitive way. Compared with findings from research in the UK and other western countries, our respondents reported more frequent symptoms of infections and higher and earlier help-seeking from a medical doctor following an infection..$^{55}$ These variations may not be solely explained by differences in pathogens and 


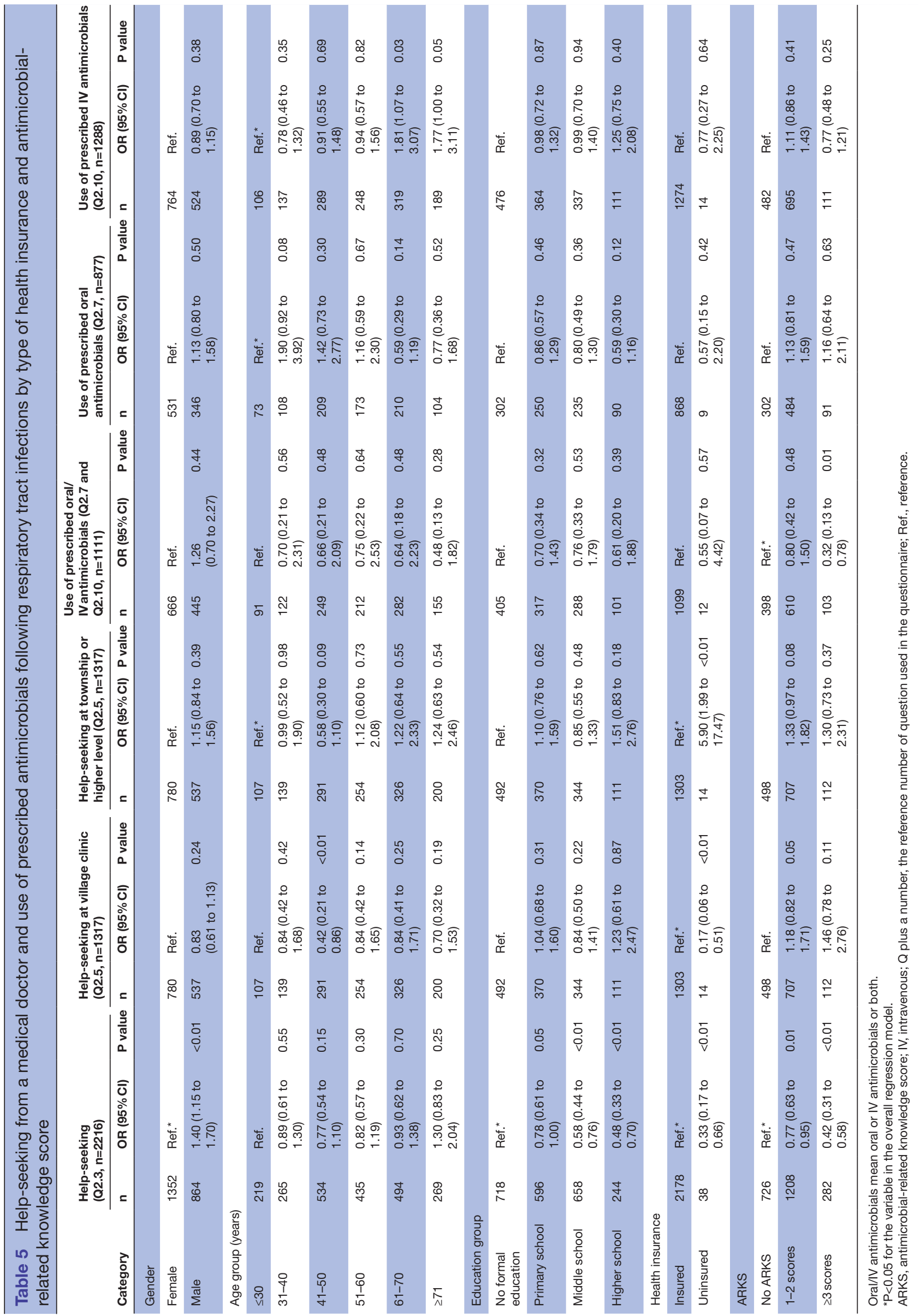


host immunity between nations. Rather, China's strong culture of collectivism may have played an important role which requires each family member to be sensitive to the health of other members and urges the sick to seek help from a doctor as soon as possible. ${ }^{56}$ In addition, the relatively low help-seeking from a medical doctor for UTIs compared with that for ARTIs and GTIs may also be partly attributable to conservative values about sex, and consequently help-seeking for related conditions, held by Chinese, especially those in rural areas. ${ }^{57}$

\section{Strengths and limitations of the study}

The study has both strengths and limitations. It is the first survey that collected data from healthcare users via a household survey while most of the existent research on antimicrobial use in China uses data from medical records or reports by medical caregivers who may be incentivised to omit recording overuse or misuse of antimicrobials so as to meet relevant policy requirements. ${ }^{58}$ As healthcare receivers, household members may be free from these concerns and hence more willing to report antimicrobial use. However, self-reported antimicrobial use may be prone to bias due, for example, to recall issues particularly among the elderly, inability to distinguish antimicrobials from other drugs which may be an issue among the less-well educated and over or under reporting by the respondents for reasons such as perceived expectations from the researchers. The study asked respondents to recall symptomatic ARTIs in the past year but GTIs and UTIs in the past 3 months. Hence, more episodes of minor ARTIs than GTIs/UTIs may not have been recalled by respondents. In addition, readers should be aware of potential selection biases. The study allowed field data collectors to exclude the household members who were 'unable to answer the questionnaire'. This may result in under recruitment of senior and illiterate residents.

\section{Next steps and future research required}

Excessive prescribing of antimicrobials in the studied primary care settings in rural Anhui is prevalent despite China's special rectification programme and health systems reforms aimed at tackling the problem. There is a clear need to target existing interventions at primary care level and to develop bespoke, community-appropriate interventions if we are to reduce antimicrobial prescribing in China. Further work is under way to identify pathways for optimising antimicrobial use in rural Anhui and China via multidisciplinary qualitative research with specific attention on its key social, cultural, economic, clinical, health systems, behavioural and other determinants to help inform future interventions.

Acknowledgements We are grateful to Anhui Provincial Commission for Health and Family Planning for their support and coordination in this application for the funding of this research and in implementing the research, especially recruiting the study sites. We also express our full acknowledgement of the rural residents who have provided advice on our research and questionnaire design.

Contributors JCha and DBW participated in conceptualising the study. JCha directed study implementation and data collection, conducted data analysis and drafted the manuscript. DBW supervised the study and together with ZH provided expertise for overall design of the study. CC, JChe, 10 and AK advised on study design, development of data collection materials and data analysis and together with DBW, JCha and ZH contributed to revising and finalising the manuscript.

Funding This study was supported by the UK-China Strategic Prosperity Fund (grant number: PPY CHN 1590/15SS19).

Competing interests None declared.

Patient consent for publication Not required.

Ethics approval The study protocol was approved by the Biomedical Ethics Committee of Anhui Medical University (reference number: 20150080).

Provenance and peer review Not commissioned; externally peer reviewed.

Data sharing statement The data sets generated and/or analysed during the current study are available from the corresponding authors on reasonable request.

Open access This is an open access article distributed in accordance with the Creative Commons Attribution Non Commercial (CC BY-NC 4.0) license, which permits others to distribute, remix, adapt, build upon this work non-commercially, and license their derivative works on different terms, provided the original work is properly cited, appropriate credit is given, any changes made indicated, and the use is non-commercial. See: http://creativecommons.org/licenses/by-nc/4.0/.

\section{REFERENCES}

1. O'Neill J. Tackling Drug-Reinstating Infections Globally: Review on Antimicrobial Resistance. 2016 http://amr-review.org (accessed on 25 February, 2016)

2. Costelloe C, Metcalfe C, Lovering A, et al. Effect of antibiotic prescribing in primary care on antimicrobial resistance in individual patients: systematic review and meta-analysis. BMJ 2010;340:c2096.

3. McGowan JE. Antimicrobial resistance in hospital organisms and its relation to antibiotic use. Rev Infect Dis 1983;5:1033-48.

4. Turnidge J, Christiansen K. Antibiotic use and resistance--proving the obvious. Lancet 2005;365:548-9.

5. Levy SB, Marshall B. Antibacterial resistance worldwide: causes, challenges and responses. Nat Med 2004;10(12 Suppl):S122-S129.

6. Shibl AM, Memish Z, Osoba A. Antibiotic resistance in developing countries. J Chemother 2001;13 Suppl 1(Suppl 1):40-4.

7. Nasrin D, Collignon PJ, Roberts L, et al. Effect of beta lactam antibiotic use in children on pneumococcal resistance to penicillin: prospective cohort study. BMJ 2002;324:28-30.

8. Ferech M, Coenen S, Malhotra-Kumar S, et al. European Surveillance of Antimicrobial Consumption (ESAC): outpatient antibiotic use in Europe. J Antimicrob Chemother 2006;58:401-7.

9. West JV. Acute upper airway infections. Br Med Bull 2002;61:215-30.

10. Guven GS, Uzun O. Principles of good use of antibiotics in hospitals. $J$ Hosp Infect 2003;53:91-6.

11. Cizman M. The use and resistance to antibiotics in the community. Int J Antimicrob Agents 2003;21:297-307.

12. Harnden A, Perera R, Brueggemann AB, et al. Respiratory infections for which general practitioners consider prescribing an antibiotic: a prospective study. Arch Dis Child 2007;92:594-7.

13. Goossens H, Ferech M, Vander Stichele R, et al. Outpatient antibiotic use in Europe and association with resistance: a cross-national database study. Lancet 2005;365:579-87.

14. Watson RL, Dowell SF, Jayaraman M, et al. Antimicrobial use for pediatric upper respiratory infections: reported practice, actual practice, and parent beliefs. Pediatrics 1999;104:1251-7.

15. McCaig LF, Besser RE, Hughes JM. Trends in antimicrobial prescribing rates for children and adolescents. JAMA 2002;287:3096-102.

16. Yin X, Song F, Gong $Y$, et al. A systematic review of antibiotic utilization in China. J Antimicrob Chemother 2013;68:2445-52.

17. Zhang $\mathrm{HJ}$, Zhang $\mathrm{YH}$, Wang $\mathrm{Y}$, et al. A retrospective study on clinical antibiotics use by 1453 inpatients. Chinese Journal of Nosocomiology 2009;2:193-5.

18. Shen ZJ. Investigation on antimicrobials use in a hospital from 2007 to 2009. Chinese Journal of Nosocomiology 2010;12:1774-5.

19. Zhou YQ, Wei P. Antibiotic use 21360 Outpatient prescriptions. Evaluation and Analysis of Drug-use in Hospitals of China 2014;5:017

20. Fan YF, Yan ML, Cai J. Review of antibiotics use by outpatients in our hospital. Evaluation and Analysis of Drug-use in Hospitals of China 2010;3:204-6.

21. Long ZH. Survey on antibiotics use of 6921 inpatients. Chin J Mod Drug Appl 2011;5:51-2. 
22. Yin J. Study on drugs use in rural areas of Shandong and Ningxia provinces. Shandong University, Jinan, China 2009:26-7.

23. Dong L, Yan H, Wang D. Antibiotic prescribing patterns in village health clinics across 10 provinces of Western China. $J$ Antimicrob Chemother 2008;62:410-5.

24. Zhao LB, Sun Q, Cheng L. Attitudes and practices of physicians and patients about antibiotics use. Chinese Health Policy 2013;6:48-52.

25. Yezli S, Li H. Antibiotic resistance amongst healthcare-associated pathogens in China. Int J Antimicrob Agents 2012;40:389-97.

26. Zhang R, Eggleston $\mathrm{K}$, Rotimi V, et al. Antibiotic resistance as a global threat: evidence from China, Kuwait and the United States. Global Health 2006;2:6.

27. Wang H, Chen M; China Nosocomial Pathogens Resistance Surveillance Study Group. Surveillance for antimicrobial resistance among clinical isolates of gram-negative bacteria from intensive care unit patients in China, 1996 to 2002. Diagn Microbiol Infect Dis 2005;51:201-8

28. Liu X, Yang X, San B, et al. Surveillance of antimicrobial resistance of clinical isolates in Kunming in 2001. Chin J Infect Chemother 2004;04:202-5.

29. Shen Z, Sun B, Du T, et al. A surveillance study on antimicrobial resistance of clinical isolates from the tertiary hospital in Hubei area. Chin J Infect Chemother 2004;05:263-7.

30. Wang F, Zhu DM, Fp H, et al. CHINET 2009 surveillance of bacterial resistance in China. Chin J Infect Chemother 2010;10:325-34.

31. Yang $\mathrm{Q}$, Wang $\mathrm{H}$, Chen $\mathrm{M}$, et al. Surveillance of antimicrobial susceptibility of aerobic and facultative Gram-negative bacilli isolated from patients with intra-abdominal infections in China: the 2002-2009 Study for Monitoring Antimicrobial Resistance Trends (SMART). Int $J$ Antimicrob Agents 2010;36:507-12.

32. Dong $F, X w X$, Song WG, et al. The changing pattern and antibiotic resistance of clinical bacterial isolates in a pediatric clinic from 2003 to 2008. Chin J Infect Chemother 2009;06:440-5.

33. Currie J, Lin W, Meng J. Addressing Antibiotic Abuse in China: An Experimental Audit Study. J Dev Econ 2014;110:39-51.

34. Reynolds L, McKee M. Factors influencing antibiotic prescribing in China: an exploratory analysis. Health Policy 2009;90:32-6.

35. Sun Q, Dyar OJ, Zhao L, et al. Overuse of antibiotics for the common cold - attitudes and behaviors among doctors in rural areas of Shandong Province, China. BMC Pharmacol Toxicol 2015;16:6.

36. Ym L, Jin Y, Liu HX, et al. Community Doctors' Clinical Usage of Antibiotics and Analysis of Influencing Factors. Chinese General Practice 2014;17:3762-5.

37. Mao W, Vu H, Xie Z, et al. Systematic review on irrational use of medicines in China and Vietnam. PLoS One 2015;10:e0117710.

38. Tomson G, Vlad I. Strengthening the rational use of drugs: International perspective and its implications for China. Chinese $J$ Health Policy 2012;5:6-9.

39. Ebert SC. Factors contributing to excessive antimicrobial prescribing Pharmacotherapy 2007;27(10 Pt 2):126S-30.

40. National Health and Family Planning Commission of the People's Republic of China. Statistical bulletin of health development in China in. 2009 http://www.moh.gov.cn/zwgkzt/pgb/201006/47783.shtml (accessed on 13 June, 2010).

41. National Health and Family Planning Commission of the People's Republic of China. Notice on national special rectification program of the clinical antimicrobials use. http://www.moh.gov.cn/mohyzs/ s3585/201305/6042979f05cf49609e96410d7314ecae.shtm (accessed on 19 April, 2011).
42. National Health and Family Planning Commission of the People's Republic of China. The stewardship strategies for the clinical antimicrobials use. http://www.nhfpc.gov.cn/fzs/s3576/201205/ 2f773c2ddbd84e19aab0b4b2d9741900.shtml (accessed on 8 May, 2012).

43. National Health and Family Planning Commission of the People's Republic of China. Notice on the further special rectification program of the clinical antimicrobials use. http://www.moh.gov.cn/ mohyzs/s3585/201305/6042979f05cf49609e96410d7314ecae.shtml (accessed on 7 May, 2013).

44. Hou D, Wang Q, Jiang C, et al. Evaluation of the short-term effects of antimicrobial stewardship in the intensive care unit at a tertiary hospital in China. PLoS One 2014;9:e101447.

45. $\mathrm{Bq} \mathrm{H}$. Effect of special rectification program of inpatients antimicrobials use. Henan Journal of Preventive Medicine 2015;26:241-3.

46. Shu W, Ren SH, Jiang J, et al. Antibiotics use in five hospitals in our district before and after the implementation of the stewardship strategies for the clinical antimicrobials use. Chinese pharmacy 2015;24:2314-7.

47. Chao J, Gu J, Zhang $\mathrm{H}$, et al. The Impact of the National Essential Medicines Policy on Rational Drug Use in Primary Care Institutions in Jiangsu Province of China. Iran J Public Health 2018;47:24-32.

48. Xiao Y, Wang J, Shen P, et al. Retrospective survey of the efficacy of mandatory implementation of the Essential Medicine Policy in the primary healthcare setting in China: failure to promote the rational use of antibiotics in clinics. Int $J$ Antimicrob Agents 2016;48:409-14.

49. Brauer R, Ruigómez A, Downey G, et al. Prevalence of antibiotic use: a comparison across various European health care data sources. Pharmacoepidemiol Drug Saf 2016;25(Suppl. 1):11-20.

50. National Bureau of Statistics of the People's Republic of China. Bulletin of the sixth national population census data of 2010 in Anhui Province. http://www.stats.gov.cn/tjsj/tjgb/rkpcgb/dfrkpcgb/201202/ t20120228_30380.html (accessed on 17 May, 2011).

51. Wang J, Wang P, Wang X, et al. Use and prescription of antibiotics in primary health care settings in China. JAMA Intern Med 2014;174:1914-20.

52. Grigoryan L, Haaijer-Ruskamp FM, Burgerhof JG, et al. Selfmedication with antimicrobial drugs in Europe. Emerg Infect Dis 2006;12:452-9

53. Bi P, Tong S, Parton KA. Family self-medication and antibiotics abuse for children and juveniles in a Chinese city. Soc Sci Med 2000;50:1445-50.

54. Butler CC, Hood K, Verheij T, et al. Variation in antibiotic prescribing and its impact on recovery in patients with acute cough in primary care: prospective study in 13 countries. BMJ 2009;338:b2242.

55. Wong CK, Liu Z, Butler CC, et al. Help-seeking and antibiotic prescribing for acute cough in a Chinese primary care population: a prospective multicentre observational study. NPJ Prim Care Respir Med 2016;26:15080.

56. Triandis HC. Individualism-Collectivism and Personality. J Pers 2001;69:907-24.

57. Chai J, Wang DB, Zhou M, et al. Developing and piloting an expert system for better routine voluntary HIV counseling and testing. AIDS Care 2012;24:424-33

58. Liu C, Yin AH, Yin C. Research on the application status and strategic of antibiotics in China. Chinese Health Service Management 2016;33:682-4. 